\title{
Superior survival with surgery for early- versus late-stage heart disease: Cause and effect or methodologic quirk?
}

\author{
Anelechi C. Anyanwu, MD
}

\author{
From the Department of Cardiovascular Surgery, Mount Sinai Medical Center, New York, NY. \\ Disclosures: Author has nothing to disclose with regard to commercial support. \\ Received for publication May 5, 2016; accepted for publication May 6, 2016. \\ Address for reprints: Anelechi C. Anyanwu, MD, Department of Cardiovascular Surgery, Mount Sinai Medical \\ Center, 1190, Fifth Ave, New York, NY 10029 (E-mail: anelechi.anyanwu@mountsinai.org). \\ J Thorac Cardiovasc Surg 2016;152:401-5 \\ $0022-5223 / \$ 36.00$ \\ Copyright (c) 2016 by The American Association for Thoracic Surgery \\ http://dx.doi.org/10.1016/j.jtcvs.2016.05.035
}

It is generally held that detection and prompt treatment of early-stage disease can prevent morbidity and mortality associated with advanced stages of disease. This reasoning forms the basis for cancer screening, the hypothesis being that deaths are prevented by detection and treatment of early-stage cancers. However, a critical review of trials on screening for neoplastic and cardiovascular disease in asymptomatic adults showed that reductions in diseasespecific mortality are uncommon, and reductions in allcause mortality are very uncommon or nonexistent with systematic application of screening tests. ${ }^{1}$ In cardiovascular medicine there are no screening trials that inform on the benefit of early intervention for asymptomatic heart disease. Physicians rely on observational studies for guidance.

A commonly applied study method is comparative analysis of outcomes of patients who were in an early stage of disease when they underwent heart surgery and patients who were at a later stage of disease at time of surgery. One such analysis was conducted by Yang and colleagues, who retrospectively evaluated the influence of preoperative pulmonary hypertension on long-term outcomes of patients who had surgery for mitral valve stenosis over a 22-year period. Not surprisingly, the long-term survival was worse in patients who had moderate or severe pulmonary hypertension at time of surgery, compared with those who did not. Based on this finding the authors recommend early surgery for severe mitral stenosis before onset of moderate pulmonary hypertension. This is contradictory to current practice guidelines, which do not recommend surgery until pulmonary hypertension becomes severe, or symptoms become pronounced. ${ }^{3}$ Yang and colleagues ${ }^{2}$ suggest that early surgery is necessary to "forestall further worsening of the pulmonary hypertension and improve long-term survival." Is this a valid inference that can be drawn from their analysis? Does superior survival seen with surgery in earlystage disease imply that operating on early-stage disease forestalls advanced disease and improves survival? The nonsurgical literature does not seem to support the concept of improved cardiovascular survival with intervention for early-stage structural heart disease. A randomized trial of

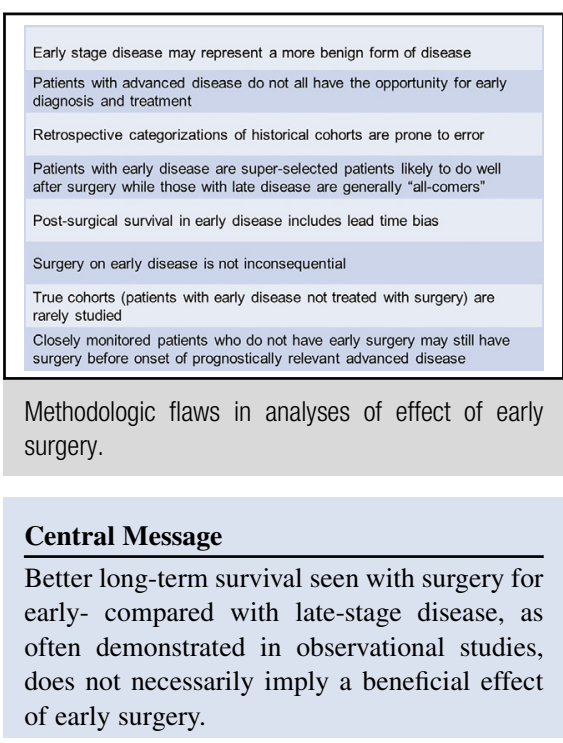

See Article page 394.

See Editorial page 302.

more than 6000 asymptomatic patients who received either a screening echocardiogram or standard primary care found no survival benefit (of screening echocardiography) at 15 years' follow-up, despite findings of structural heart disease in $7 \%$ of those who had screening echocardiograms. ${ }^{4}$ Why then does the surgical literature suggest that early detection and treatment of structural heart disease should result in improved survival? An appreciation of the methodologic nuances that underlie comparisons of surgical interventions according to stage of disease is critical to understanding and interpreting such studies (Figure 1).

\section{VARIABLE NATURAL HISTORY}

Analyses of outcomes of intervention on early versus late disease have an inherent presumption that the disease is a spectrum through which patients progress sequentially at a similar rate from no disease through subclinical, mild, moderate, and advanced stages of disease, the inference being that all patients with advanced disease were once at a detectable subclinical or mild stage of disease that would have lent itself to surgical intervention. However, not all patients follow such a disease trajectory. For some patients with cardiac disease, the first detection of disease may be at a severe symptomatic state, so there may never have been an opportunity for intervention at an earlier stage. 


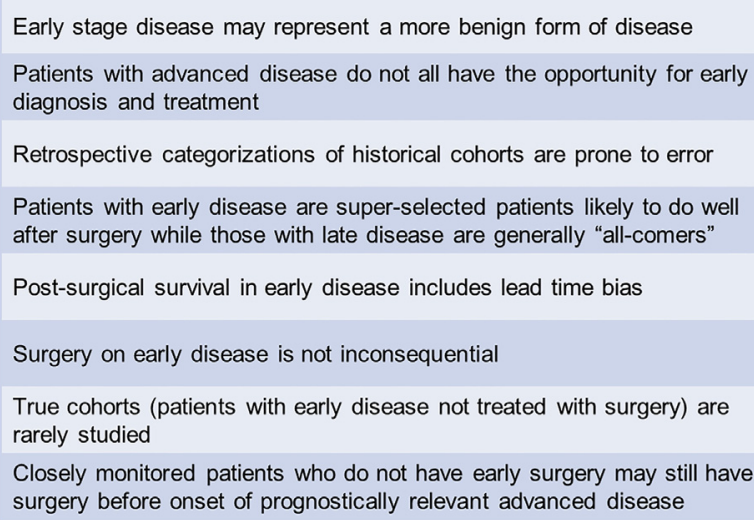

FIGURE 1. Methodologic problems with comparing survival of patients who had surgery in early stages of disease compared with those operated in late stage of disease.

Therefore, although in theory earlier intervention might have yielded better outcomes, in reality some patients may not have had the opportunity for early treatment because the diagnosis may not have been possible at that stage, or they might not have spent sufficient time in an early phase to allow therapy. Yang and colleagues ${ }^{2}$ do not present data on the interval from diagnosis of severe mitral stenosis to surgery, but notably the average age was similar in patients who had none, mild, moderate, and severe pulmonary hypertension. This raises question as to whether those with severe pulmonary hypertension represented a subset of more rapid progression of stenosis (as otherwise one would expect an older age in those with severe pulmonary hypertension because they should have spent a longer time in a diseased state). Because disease progresses at different rates, some patients who present with advanced disease may not have been in an early stage for a sufficiently long period that would have allowed possibility of earlier intervention. This phenomenon of so-called length time bias exaggerates the benefit of intervention on early-stage disease because patients treated at an earlier stage are more likely to have had a long latent phase of early disease, and therefore invariably do better compared with those presenting already in an advanced phase of disease who may have a more rapidly progressing form of disease and generally do worse. Therefore, patients who lend themselves to the option of early surgery may represent a more indolent form of disease and may not be similar to those who undergo surgery at a more advanced stage of disease.

\section{RETROSPECTIVE CODING OF PROCEDURES MAY BE FLAWED}

Surgical series evaluating long-term outcomes will, necessarily, include patients operated on in a historical era, some series going back as far as the 1980s. Yang and colleagues $^{2}$ studied patients who had surgery for mitral stenosis between 1992 and 2014. When such long time periods are studied, it is invariable that the clinical understanding, diagnosis, and management of disease would have undergone evolution over the course of the study. Furthermore, the more distant a procedure date is from the current era, the less reliable the data (based on current understanding and definitions). In the report by Yang and colleagues, ${ }^{2}$ as in many other surgical series, patient inclusion was determined by retrospective review of operative reports. Such an approach relies on the assumption that categorizations in operative reports are accurate. However, it is well recognized that operative reports are prone to errors and inaccuracies, particularly where there has been a delay in writing or dictating the report, or where reports are dictated by resident physicians. ${ }^{5}$ Defining whether patients meet inclusion criteria can also be problematic. The authors report that they did not utilize diagnostic data to confirm the severity of mitral stenosis (because this was not available on all cases), but rather assumed that the patient had severe stenosis if the surgeon reported mitral stenosis as the indication for surgery. ${ }^{2}$ However, only objective measurement by echocardiography or cardiac catheterization can confirm that a patient indeed has severe mitral stenosis. Yang and colleagues ${ }^{2}$ did not provide any objective preoperative measurements of valve function, such as valve area or gradient, so it is possible that not all patients in the study had severe mitral stenosis, and that some patients who had surgery for severe mitral stenosis were not included in the study because the surgeon had not documented the indication correctly.

Of note, $10 \%$ of patients undergoing operation for severe mitral stenosis in the study by Yang and colleagues ${ }^{2}$ had normal pulmonary artery pressure, and two-thirds of those with normal pulmonary pressures were reported to be in New York Heart Association class III or IV. It is unexpected that patients with severely symptomatic mitral stenosis would have normal pulmonary artery pressure, because pulmonary artery hypertension is typically a requisite component of the pathophysiology of symptoms in mitral valve stenosis. ${ }^{6}$ Furthermore, as surgical therapy has not been recommended for minimally symptomatic mitral stenosis without severe pulmonary hypertension in current and previous practice guidelines, ${ }^{3,7,8}$ it should have been unusual for such patients to undergo mitral valve surgery for mitral stenosis in the 1990s and 2000s. This raises the question as to whether there could have been some miscoding of some of these patients reported to have severe stenosis with normal pulmonary artery pressure. Indeed, the authors ${ }^{2}$ excluded $24 \%$ of patients with mitral stenosis undergoing operation during the study period because the pulmonary artery pressure was undocumented or unknown. It is extremely unlikely, even in 1992, that 1 in 4 patients undergoing mitral valve surgery for stenosis did not have a measurement of pulmonary pressure, so this raises questions as to the robustness, accuracy, and 
completeness of the pulmonary hemodynamic data. In a retrospective study, such coding errors are inevitable and often cannot be reconciled because the most important repository of data-the patient at the time point of intervention-is not available for confirmatory review.

\section{TRUE DENOMINATORS ARE UNCERTAIN}

A surgical series invariably only accounts for patients with a disease who were referred to a surgeon, accepted for surgery, and included in the study. Patients who, for whatever reason, are not referred to surgeons, are not accepted for surgery, or who after acceptance for surgery are not included in a study cohort, are not reported. Where conventional indications for intervention do not mandate surgery for early stages of a disease, patients with earlystage disease undergoing surgery will invariably be a super-selected subset of the entire population of patients with early disease. Typically, those seen as highly likely to have a good outcome with surgery (mainly young patients or older patients with minimal comorbidity) would be preferentially selected for early intervention. This selection often transforms to excellent surgical outcomes for most surgical procedures done in the setting of early disease. These excellent results may give the erroneous impression that surgery on early-stage disease saves lives. For example, in a study comparing surgery with medical surveillance for asymptomatic mitral regurgitation, ${ }^{9}$ the 7year event-free survival for asymptomatic patients who had mitral valve repair was $99 \%$, compared with $85 \%$ in patients treated medically. A $99 \%$ midterm event-free survival would be unprecedented for any form of surgery for acquired heart disease and almost certainly implies heavily biased patient selection, restrictive study inclusion criteria, data inaccuracies or miscoding, or unknown sources of bias or error. Indeed, when the same investigators performed a propensity-based analysis several years later, ${ }^{10}$ the difference in event-free survival between early surgery and medical surveillance was less pronounced.

In the study by Yang and colleagues, ${ }^{2}$ it is not known the criteria by which the patients with normal or mildly elevated pulmonary artery pressures were selected for surgery (as opposed to alternative treatment approach), nor the denominator from which they were selected. With mitral stenosis, the confounding effect of alternative therapies is important because patients with severe stenosis may be treated with balloon valvuloplasty as initial or alternative therapy. Yang and colleagues ${ }^{2}$ did not provide a denominator that included patients undergoing percutaneous valvuloplasty, making it impossible for readers to characterize the selection process.

\section{LEAD TIME BIAS}

Comparing outcomes of surgery on patients with earlystage heart disease with those operated at later stage of disease will invariably show better postsurgical outcomes in those with early disease. This is partly due to the phenomenon of lead-time bias in which excellent survival after surgery for subclinical or mild disease gives the erroneous impression that surgery has prolonged survival in those patients compared to if they had been operated at later stages of disease; in reality, the survival ascribed to surgery includes a period of survival (with latent phase of disease) that would likely have still occurred had surgery not been performed. For example, early institution of extracorporeal membrane oxygenation (ECMO) in patients with early respiratory failure and moderate ventilator demands will yield much superior post-ECMO survival when compared with ECMO applied solely for refractory respiratory failure. Although this may give impression that early institution of ECMO saves lives, the reality is that many of the patients who receive early ECMO would have survived regardless of whether ECMO had been instituted, making it impossible to define the true benefit of early intervention.

Only randomization or true case-control matching can exclude the bias of lead time. A recent analysis by Coutinho and colleagues ${ }^{11}$ of patients undergoing mitral valve surgery for bileaflet or anterior leaflet prolapse found that patients with asymptomatic or minimally symptomatic disease had a higher rate of valve repair and superior long-term survival after mitral valve surgery compared with patients who were in New York Heart Association class III or IV (10-year survival $85 \%$ vs $64 \%$; 20 -year survival $76 \%$ vs $17 \%$ ), leading the authors to conclude that asymptomatic and minimally symptomatic patients had the greatest benefit from valve repair. However, the likelihood is that a majority of the asymptomatic patients would have had similar, or only mildly reduced, survival had they not had early surgery, and it may not necessarily be the early surgical intervention that resulted in the good long-term survival, but rather patients experienced the expected survival for the stage of disease regardless of early intervention. Indeed, observational data suggest that the survival advantage of early surgery over medical surveillance for asymptomatic mitral regurgitation is modest-likely no more than $5 \%$ to $10 \%$ at 10 years ${ }^{10,12}$ — so early surgery alone could not be the cause of the large survival differential in the symptomatic versus minimally symptomatic patients reported by Coutinho and colleagues. ${ }^{11}$ Lead-time bias was likely a major factor in differential survival seen with surgery on early compared with advanced stages of valve disease in that study, and possibly also played a role in the survival differential reported by Yang and colleagues. ${ }^{2}$

\section{SURGERY ON EARLY DISEASE IS NOT INCONSEQUENTIAL}

An implicit assumption in most analyses of early surgery is that surgery results in elimination of the cardiac risk in patients with early-stage disease without long-term 
consequence. In reality, patients who undergo successful cardiac surgery will not necessarily be disease-free in the long-term because: (1) the presence of a prosthetic valve or a repaired valve or defect is rarely physiologically normal and places the patient at risk for both pathologic and physiologic sequelae; (2) the effects of the original disease on the heart may not totally reverse even with correction (eg, ventricular dysfunction, scarring, or irritability could persist after correction of a structural defect); and (3) surgery on the heart, even if uncomplicated, may itself have deleterious long-term effects in some patients. There remains no ideal treatment for diseased heart valves. All replacement options are associated with reduced life expectancy and valve-related morbidity. Valve repair is also associated with incremental morbidity (although to a substantially lesser degree than replacement) and has with a linear failure rate of up to $1 \%$ to $5 \%$ per year, depending on the etiology. ${ }^{13}$

Considering that a substantial proportion of stenotic rheumatic valves treated surgically are replaced $(80 \%$ in study by Yang et $\mathrm{al}^{2}$ ), it cannot be certain that early surgical intervention for rheumatic mitral stenosis will improve survival as mitral valve replacement is associated with substantial incidence of late mortality. Indeed, liberal application of valve replacement in early-stage mitral stenosis could paradoxically yield worse long-term survival if valve prosthesis-related mortality exceeds that of untreated earlystage mitral stenosis. Additionally, surgical intervention can unfavorably alter the natural history of early disease. In oncology, some experts argue that surgery on early cancer may result in disruption of the natural history of previously undisturbed disease with resultant acceleration of disease progression. ${ }^{14} \mathrm{~A}$ similar effect is theoretically possible for heart valve disease. For example, leaflet scarring, fibrosis, and pannus formation are relatively common mechanisms of failure after surgical valvuloplasty or replacement, ${ }^{15}$ but do not typically occur in de novo nonrheumatic valves. Although a caveat of ultra-low surgical mortality should be met for surgery on asymptomatic disease, ${ }^{3}$ it cannot be assumed that because patients survive surgery without complications that they have not experienced harm.

\section{ABSENCE OF TRUE CONTROLS}

Observational studies do not inform robustly on the effect of early surgery partly because of the invariable selection that takes place to arrive at a population of patients chosen for early intervention. To reliably make a comparison of early versus late surgery, one needs to compare a cohort of patients who underwent early surgery with a matched group of patients with similar disease who did not undergo early surgery. Such comparisons are rare. Of note, Yang and colleagues ${ }^{2}$ did not include a control group of patients with early severe mitral stenosis who were not treated by surgery.
Some authors have attempted propensity matching of surgical patients with patients managed medically, but this does not balance for a substantial amount of selection bias because several key determinants of the selection process are not controlled for in propensity modeling. ${ }^{16}$ The limitations of observational studies in defining the effect of early surgery versus delayed surgery or medical surveillance are highlighted by the conflicting results of studies comparing surgery with medical surveillance in patients with asymptomatic (degenerative) mitral regurgitation..$^{9,10,12,17,18}$ The explanation for the seemingly discordant results likely lies in differences in patient cohorts, patient selection criteria, methods of medical and surgical management, and the absence of truly matched controls.

Furthermore, an assumption inherent in most observational studies of early versus late surgery is that there is dichotomous separation between the 2 strategies, and that when a patient does not have early surgery, surgery will not take place until the disease is at an advanced stage. In practice, there is a wide spectrum of disease presentation and management, such that late surgery is not the inevitable alternative to early surgery in early stage heart disease. There is a middle ground-patients who do not have early surgery may still undergo surgery before the disease progresses to an advanced stage. For example, a strategy of close medical surveillance (ie, watchful waiting) of patients with asymptomatic mitral regurgitation allows prompt referral to surgery when patients develop prespecified triggers-this approach was demonstrated in a single study ${ }^{17}$ to have midterm outcomes similar to that expected with early surgery.

Although comparisons of patients undergoing operation at early versus late stages of disease may provide some insightful data, the results are very predictable, and almost invariably suggest early surgery results in better patient survival. An observation of superior survival in patients who underwent surgery at earlier (compared with later) stage of disease reflects more the limitations of the underlying methodology rather than a definite beneficial effect of early surgery on long-term survival. Only a randomized trial can truly balance for the selection and methodologic bias inherent in such studies and provide robust data on the effect of early surgical intervention for early-stage structural heart disease.

\section{References}

1. Saquib N, Saquib J, Ioannidis JP. Does screening for disease save lives in asymptomatic adults? Systematic review of meta-analyses and randomized trials. Int $J$ Epidemiol. 2015;44:264-77.

2. Yang B, DeBenedictus C, Watt T, Farley S, Salita A, Hornsby W, et al. The impact of concomitant pulmonary hypertension on early and late outcomes following surgery for mitral stenosis. J Thorac Cardiovasc Surg. 2016;152:394-400.e1.

3. Nishimura RA, Otto CM, Bonow RO, Carabello BA, Erwin JP III, Guyton RA, et al. 2014 AHA/ACC guideline for the management of patients with valvular heart disease: A report of the American College of Cardiology/American Heart 
Association Task Force on Practice Guidelines. J Thorac Cardiovasc Surg. 2014; 148:e1-132.

4. Lindekleiv H, Lochen ML, Mathiesen EB, Njolstad I, Wilsgaard T, Schirmer H. Echocardiographic screening of the general population and long-term survival: a randomized clinical study. JAMA Intern Med. 2013;173:1592-8.

5. Novitsky YW, Sing RF, Kercher KW, Griffo ML, Matthews BD, Heniford BT. Prospective, blinded evaluation of accuracy of operative reports dictated by surgical residents. Am Surg. 2005;71:627-31.

6. Chandrashekhar Y, Westaby S, Narula J. Mitral stenosis. Lancet. 2009;374: 1271-83.

7. Bonow RO, Carabello B, de Leon AC, Edmunds LH Jr, Fedderly BJ, Freed MD, et al. ACC/AHA guidelines for the management of patients with valvular heart disease. Executive summary. A report of the American College of Cardiology/ American Heart Association Task Force on Practice Guidelines (Committee on Management of Patients With Valvular Heart Disease). J Heart Valve Dis. 1998;7:672-707.

8. Bonow RO, Carabello BA, Kanu C, de LA Jr, Faxon DP, Freed MD, et al. ACC/ AHA 2006 guidelines for the management of patients with valvular heart disease: a report of the American College of Cardiology/American Heart Association Task Force on Practice Guidelines (writing committee to revise the 1998 Guidelines for the Management of Patients With Valvular Heart Disease): developed in collaboration with the Society of Cardiovascular Anesthesiologists: endorsed by the Society for Cardiovascular Angiography and Interventions and the Society of Thoracic Surgeons. Circulation. 2006;114:e84-231.

9. Kang DH, Kim JH, Rim JH, Kim MJ, Yun SC, Song JM, et al. Comparison of early surgery versus conventional treatment in asymptomatic severe mitral regurgitation. Circulation. 2009;119:797-804.
10. Kang DH, Park SJ, Sun BJ, Cho EJ, Kim DH, Yun SC, et al. Early surgery versus conventional treatment for asymptomatic severe mitral regurgitation: a propensity analysis. J Am Coll Cardiol. 2014;63:2398-407.

11. Coutinho GF, Correia PM, Branco C, Antunes MJ. Long-term results of mitral valve surgery for degenerative anterior leaflet or bileaflet prolapse: analysis of negative factors for repair, early and late failures, and survival dagger. Eur J Cardiothorac Surg. 2016;50:66-74.

12. Suri RM, Vanoverschelde JL, Grigioni F, Schaff HV, Tribouilloy C, Avierinos JF et al. Association between early surgical intervention vs watchful waiting and outcomes for mitral regurgitation due to flail mitral valve leaflets. JAMA. 2013;310:609-16.

13. Anyanwu AC, Adams DH. Why do mitral valve repairs fail? J Am Soc Echocardiogr. 2009;22:1265-8.

14. Baum M, Demicheli R, Hrushesky W, Retsky M. Does surgery unfavourably perturb the "natural history" of early breast cancer by accelerating the appearance of distant metastases? Eur J Cancer. 2005;41:508-15.

15. Anyanwu AC, Itagaki S, Varghese R, Castillo J, Chikwe J, Adams DH. Re-repai of the mitral valve as a primary strategy for early and late failures of mitral valve repair. Eur J Cardiothorac Surg. 2014;45:352-7.

16. Anyanwu AC. The vagaries of patient selection in cardiovascular surgery. $J$ Thorac Cardiovasc Surg. April 22, 2016 [Epub ahead of print].

17. Rosenhek R, Rader F, Klaar U, Gabriel H, Krejc M, Kalbeck D, et al. Outcome of watchful waiting in asymptomatic severe mitral regurgitation. Circulation. 2006; 113:2238-44.

18. Enriquez-Sarano M, Avierinos JF, Messika-Zeitoun D, Detaint D, Capps M Nkomo V, et al. Quantitative determinants of the outcome of asymptomatic mitral regurgitation. N Engl J Med. 2005;352:875-83. 